\title{
ESTADO NUTRICIONAL E FATORES ASSOCIADOS EM IDOSOS
}

Marta Alice Gomes Campos*, Enio Roberto Pietra Pedroso, Joel Alves lamounier, Enrico Antonio Colosimo, Marcelo Militão Abrantes

Trabalho realizado na Faculdade de Medicina da UFMG, Belo Horizonte, MG

*Correspondência:

Rua Prof. Norton Kaiserman

45/304,

Cep: $30310-570$

Anchieta, BH, MG

Tel.: (31) 9977-3041

martaalicecampos@ig.com.br

\begin{abstract}
RESUMO
Oв|Eтrvo. O presente estudo descreve o perfil nutricional e fatores associados de idosos brasileiros.

MÉTodos. Foram utilizados dados da Pesquisa sobre Padrões de Vida (PPV), realizada pelo IBGE entre 1996/1997. O Índice de Massa Corporal-IMC ( $\mathrm{kg} / \mathrm{m}^{2}$ ), indicativo do estado nutricional, é a variável resposta do presente estudo. Foi utilizada análise multivariada com regressão logística politômica.

Resultados. A prevalência geral de baixo peso foi de 5,7\%, de eutrofia 50,4\%, sobrepeso 32,3\% e obesidade II,6\%. O gênero feminino apresentou chance I,32 vez maior de sobrepeso (IC 95\% 0,99-I,74, p=0,05) e 4, I I vezes maior de obesidade (IC 95\% $2,57-6,57, p=0,00)$. O envelhecimento aumentou o risco de baixo peso e diminuiu o de sobrepeso e obesidade. As menores faixas de renda domiciliar apresentaram aumento do risco de baixo peso; por outro lado, o aumento da renda domicillar elevou o risco de sobrepeso e obesidade. Os idosos portadores de doença crônica apresentaram maior risco de alterações do estado nutricional.

Conclusão. Os resultados indicam que há maior prevalência de eutrofia e sobrepeso, e baixa prevalência de obesidade e baixo peso. As variáveis associadas com alteração do estado nutricional em relação à eutrofia foram gênero feminino, escolaridade, renda familiar, problema crônico de saúde e idade.
\end{abstract}

Unitermos: Inquéritos nutricionais. Perfis epidemiológicos. Peso corporal. Idoso.

\section{INTRODUÇÃO}

No Brasil, o processo de envelhecimento da população deve-se ao rápido declínio das taxas de mortalidade e de fecundidade. Em 2050, os idosos corresponderão a 14,2\% da população brasileira', o que sinaliza a necessidade de se conhecer mais sobre o envelhecimento, suas repercussões e impacto sobre o sistema de saúde brasileiro. A promoção da saúde pode minimizar o impacto que o envelhecimento causa ao sistema de saúde. Um dos fatores relacionados ao envelhecimento sadio é a boa nutrição durante toda a vida. $O$ estado nutricional adequado aumenta o número de pessoas que se aproximam do seu ciclo máximo de vida². Por meio da avaliação nutricional, é possível identificar indivíduos em risco nutricional aumentado para danos à sua saúde e estabelecer programas de intervenção com o objetivo de reduzi-los ${ }^{3}$

O estudo aqui apresentado avalia a prevalência de distúrbios nutricionais e de características sociodemográficas associadas na população de idosos não asilados que residem nas regiões Nordeste e Sudeste do Brasil (Regiões NE+SE) e na região metropolitana de Belo Horizonte (RMBH).

\section{Métodos}

Este trabalho utilizou dados de uma amostra representativa da população idosa brasileira, colhidos pelo Instituto Brasileiro de Geografia e Estatística (IBGE) na Pesquisa Sobre Padrões de Vida (PPV). Detalhes sobre a amostragem e motivos para a escolha das regiões NE e SE já foram publicados ${ }^{4,5}$. O IBGE disponibiliza os dados em CD-ROM, a partir do qual foram convertidos para o programa Epilnfo, onde foram manipulados.
Estão disponíveis na PPV dados sobre 1661 idosos residentes nas regiões NE e SE do Brasil. Destes, I 42 (8,5\%) não tinham informações de peso e/ou estatura e foram excluídos do estudo. Sendo assim, foram considerados um total de 1519 idosos: 73 I da região NE e 788 da região SE. Os idosos foram, então, divididos em dois grupos: idosos residentes na RMBH $(n=149)$ e o restante de idosos $(n=1370)$, denominados nesta pesquisa como regiões NE + SE.

Nesta pesquisa, a classificação nutricional foi realizada segundo as recomendações da OMS, baseadas em informações científicas sobre aumento do risco de morbidade e mortalidade ${ }^{2}$. Usou-se a definição de idoso preconizada pela OMS para países em desenvolvimento: idade igual ou maior a 60 anos $^{2}$. A região de domíiliio foi classificada como rural ou urbana, de acordo com a classificação oficial do Censo Demográfico Brasileiro ${ }^{6}$. A renda domiciliar foi calculada com base na renda proveniente do trabalho (incluindo rendimentos extras e benefícios), aluguel e outras fontes de renda de cada membro da família residente no domićlio e aferida em reais, considerado o valor do salário mínimo vigente durante a data da coleta dos dados. A escolaridade foi quantificada em anos completos de estudo concluídos com aprovação dos entrevistados que freqüentam ou já freqüentaram a escola.

A comparaçãa entre as prevalências e a verificação de associação com as diversas variáveis foram feitas por meio da comparação de proporções, usando-se o teste do Qui-quadrado e Tabelas $2 \times 2$ para variáveis categóricas binárias (dicotômicas). Quando uma das freqüências esperadas foi menor que 5, foi utilizado o teste de Fisher. Para as variáveis categóricas ordinais, foi utilizado o teste de Quiquadrado com tendência linear. A diferença de risco entre as categorias foi quantificada pelo odds ratio e intervalo de confiança. Foi considerado nível de significância de 5\%. A análise univariada foi feita 
por meio do software Epilnfo versão 6.04. A identificação de variáveis associadas com desvio do estado nutricional para além da eutrofia foi realizada separadamente para a RMBH e regiões NE + SE para identificar possíveis fatores de confusão.

Todas as variáveis com valor de $p<0,25$ na análise univariada, em qualquer uma das classificações do estado nutricional, foram incluídas no modelo inicial da análise multivariada, feita no programa MINITAB versão | I.2, utilizando a regressão logística politômica (variável dependente com mais de duas categorias). Em seguida, foram retiradas, uma a uma, as variáveis que apresentaram maior valor de $p$ até que restassem apenas variáveis com significância estatística em pelo menos uma das categorias de estado nutricional. O estado nutricional foi a variável resposta (dependente) e as demais foram variáveis independentes. Nesta análise, foram considerados apenas os idosos que apresentaram todos os dados referentes às seguintes variáveis: sexo, idade, classificação nutricional, cônjuge, local de domićlio, escolaridade, renda domiciliar, renda de aposentadoria, prática de exercício físico, problema crônico de saúde, acompanhamento médico para problema crônico de saúde, problema agudo de saúde, auto-avaliação do estado de saúde e convênio de saúde, totalizando $n=1$. 107 idosos.

Para a análise multivariada, as variáveis idade, auto-avaliação do estado de saúde e renda domiciliar foram recodificadas devido ao pequeno número de idosos em uma ou mais categorias. Para a variável idade, as categorias 80-89 e 90-99 anos foram agrupadas em uma única categoria. Para a variável auto-avaliação do estado de saúde, a categoria não informado $(n=4)$ foi excluída. Para a variável renda domiciliar, as categorias de renda $<$ I salário mínimo e de I a 2 salários mínimos foram agrupadas em uma única categoria. A variável local de domicílio foi codificada em duas categorias: idosos residentes na RMBH e idosos residentes nas regiões NE+SE excluindo a RMBH. As variáveis renda individual e dias de exercício físico por semana não entraram nos modelos de análise multivariada porque o número de idosos que responderam a estas perguntas foi muito pequeno. A variável renda de aposentadoria teve o mesmo significado da variável renda individual e entrou nos modelos de análise multivariada.

O projeto de pesquisa foi aprovado pelo Comitê de Ética em Pesquisa da UFMG - COEP, sob Parecer n ETIC I54/03.

\section{Resultados}

Neste estudo, dentre os idosos que tiveram medidas de peso e estatura aferidas, metade $(50,4 \%)$ foi classificada como eutrófica. As alterações mais significativas do estado nutricional foram encontradas em menos de $1 / 5$ dos idosos (5,7\% para baixo peso e $11,6 \%$ de obesidade). O sobrepeso foi encontrado em 32,3\% dos idosos pesquisados. Considerando a distribuição por gênero, no presente estudo, a prevalência das categorias de classificação nutricional entre homens idosos foi de 6\% para baixo peso, 56,5\% para eutrofia, 31,9\% para sobrepeso e 5,6\% para obesidade. Entre as idosas, as prevalências de classificação nutricional segundo o gênero foram de 5,4\%, 45,6\%, $32,7 \%$ e $16,3 \%$, respectivamente.

As Tabelas I e 2 apresentam os resultados da análise univariada. A Tabela I apresenta a comparação entre as classificações nutricionais baixo peso, sobrepeso e obesidade, tomando os eutróficos como categoria padrão $(O R=1,0)$ para diversas variáveis entre os idosos domiciliados nas regiões NE+SE. Observa-se que as variáveis gênero, faixa etária, área de domićlio, região demográfica, anos de estudo, exercício físico, dias de exercício físico por semana, estado de saúde, doença crônica, convênio de saúde, renda domiciliar, renda individual e renda de aposentadoria apresentaram significância estatística em pelo menos uma classificação nutricional. As variáveis cônjuge, acompanhamento médico para doença crônica e doença aguda apresentaram tendência de significância estatística $(p<0,25)$ para algum desvio da eutrofia. As variáveis motivo para praticar exercício físico, duração do exercício físico e atendimento de doença aguda não apresentaram significância estatística para nenhuma das classificações nutricionais.

A Tabela 2 apresenta os dados descritos na Tabela I para os idosos domiciliados na RMBH. Somente a variável cônjuge para os idosos com sobrepeso e a variável renda individual para os idosos portadores de baixo peso apresentaram significância estatística. Idoso que tem cônjuge apresenta 2,26 vezes mais chance de sobrepeso quando comparado ao eutrófico. Essa associação não foi encontrada no modelo final da análise multivariada. Para a renda individual, não foi possível calcular o OR porque uma das caselas apresentou valor igual a zero.

A Tabela 3 apresenta o modelo final da análise multivariada. Observa-se que, usando o sexo masculino e o estado nutricional eutrófico como padrões, as mulheres apresentaram chance 4,1 I vezes maior de obesidade ( $p=0,00$, IC 95\% 2,57-6,57) e I,32 vez maior de sobrepeso ( $p=0,05$, IC 95\% 0,99-I,74). Não houve associação entre gênero e baixo peso $(p=0,94)$.

Para a variável idade, a categoria 60 a 69 anos foi tomada como padrão. Observa-se que o aumento da idade diminui a chance de obesidade e de sobrepeso e aumenta a chance de baixo peso. Entre os indivíduos entre 70 e 79 anos, a chance de obesidade foi 0,61 vez menor (I ,63 vez mais chance de ser eutrófico) $(p=0,03$, IC $95 \% 0$, 40-0,95), 0,67 vez menor para sobrepeso ( 1,49 vez mais chance de ser eutrófico) ( $p=0,0$ I, IC 95\% 0,49-0,9I), enquanto a chance de baixo peso nesta faixa etária foi 2, I7 vezes maior $(p=0,00$, IC 95\% I,26$3,75)$. Entre os idosos com idade maior ou igual a 80 anos, a chance de obesidade foi 0,16 vez menor ( 6,25 vezes mais chance de ser eutrófico) ( $p=0,00$, IC 95\% 0,05-0,44) e 0,5 I vez menor para sobrepeso (I,96 vez mais chance de ser eutrófico) ( $p=0,00, I C 95 \% 0,32-0,83)$. Nesta faixa etária, a chance de baixo peso foi 2,16 vezes maior $(p=0,03, I C$ $95 \% 1,04-4,48)$.

Os idosos portadores de problema crônico de saúde foram tomados como categoria referência. Observa-se que idosos saudáveis (não referiram ter problema crônico de saúde) apresentaram maior chance de eutrofia. Idosos saudáveis tiveram 0,54 vez menos chance de obesidade ( 1,85 vez mais chance de ser eutrófico, $p=0,00$, IC $95 \%$ $0,36-0,81), 0,62$ vez menos chance de sobrepeso $(1,61$ vez mais chance de ser eutrófico, $p=0,00$, IC 95\% 0,47-0,81) e 0,59 vez menos chance de baixo peso $(1,69$ vez mais chance de ser eutrófico, $p=0,03$, IC 95\% 0,35-0,97).

A chance de obesidade e sobrepeso aumentou entre os idosos com maior nível de renda domiciliar, tomando como referência a categoria renda domiciliar até dois salários mínimos. Idosos com renda domiciliar entre 5 e 10 salários mínimos apresentaram 2,5 vezes mais chance de obesidade ( $p=0,03$, IC 95\% 1,07-5,86) e 2,7 vezes mais chance de sobrepeso ( $p=0,00, I C$ 95\% I,49-4,93). 


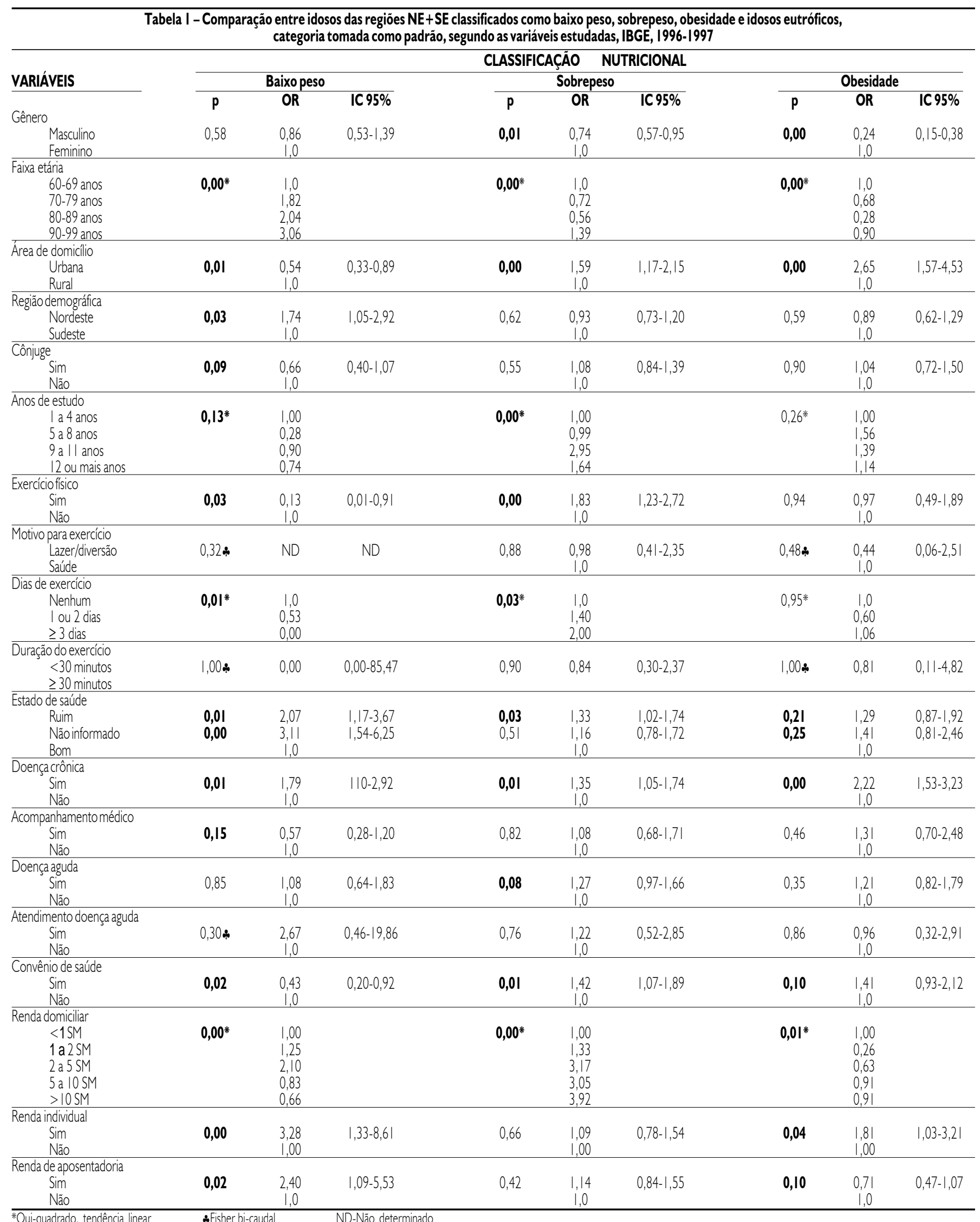


Tabela 2 - Comparação entre idosos da RMBH classificados como baixo peso, sobrepeso, obesidade e idosos eutróficos, categoria tomada como padrão, segundo as variáveis estudadas, IBGE, 1996-1997

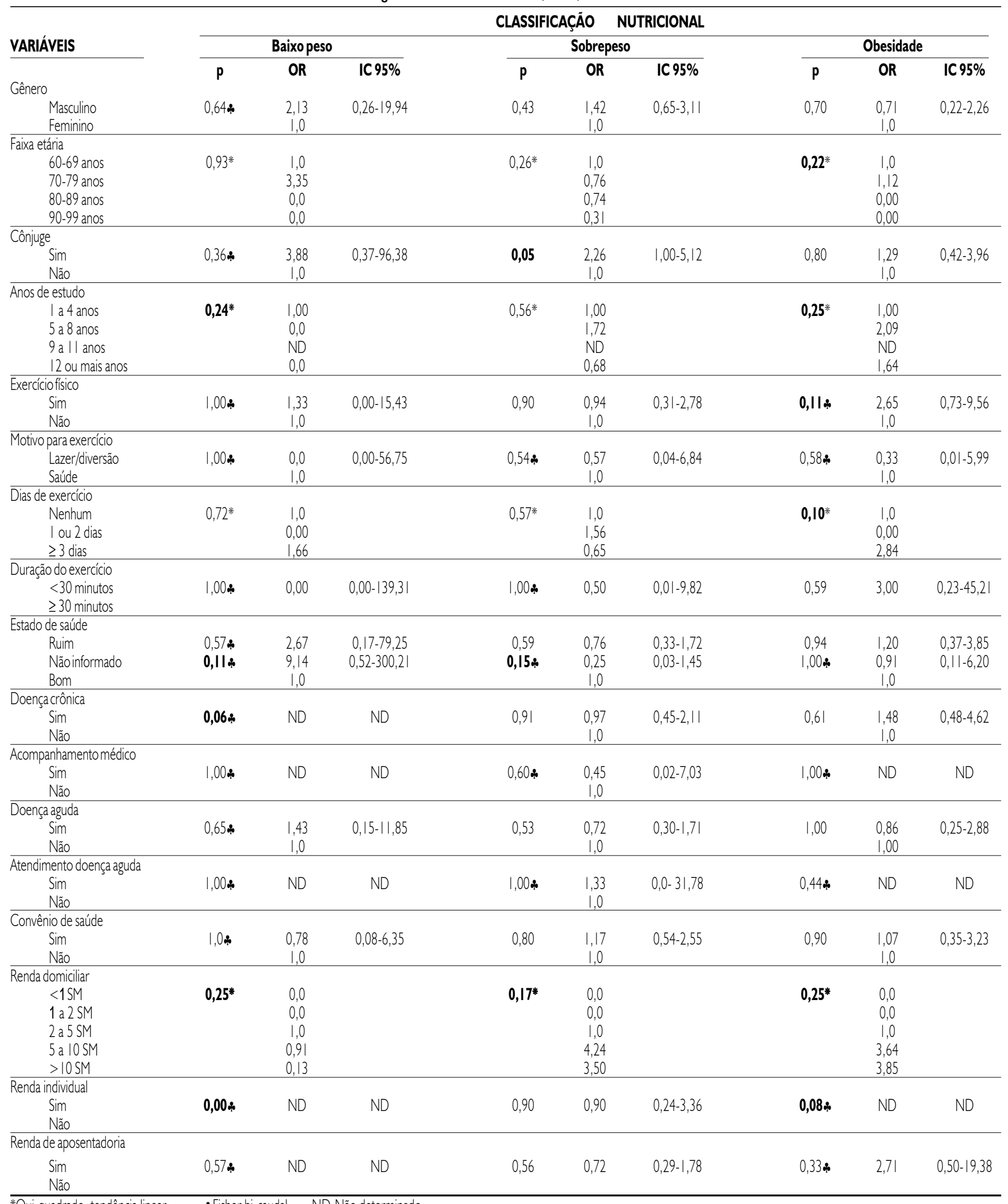

*Qui-quadrado, tendência linear *Fisher bi-caudal ND-Não determinado 
Tabela 3 - Modelo final da análise multivariada do presente estudo, IBGE, 1996-1997

\begin{tabular}{|c|c|c|c|c|c|c|c|c|c|c|c|c|}
\hline \multirow[b]{2}{*}{ VARIÁVEIS } & \multicolumn{12}{|c|}{ CLASSIFICAÇÃO } \\
\hline & \multicolumn{4}{|c|}{ BP em relação a EU } & \multicolumn{4}{|c|}{ SP em relação a EU } & \multicolumn{4}{|c|}{ OB em relação a EU } \\
\hline & Coef. & $p$ & OR & IC $95 \%$ & Coef. & $p$ & OR & IC $95 \%$ & Coef. & $p$ & OR & IC $95 \%$ \\
\hline Constante & $-1,856$ & 0,00 & & & $-0,933$ & 0,02 & & & $-3,444$ & 0,00 & & \\
\hline \multicolumn{13}{|l|}{ Sexo } \\
\hline Masculino & & & 1,00 & & & & 1,00 & & & & 1,00 & \\
\hline Feminino & 0,018 & 0,94 & 1,02 & $0,62-1,68$ & 0,274 & 0,05 & 1,32 & $0,99-1,74$ & 1,414 & 0,00 & $4, I I$ & $2,57-6,57$ \\
\hline \multicolumn{13}{|l|}{ Escolaridade } \\
\hline I a 4 anos & & & 1,00 & & & & 1,00 & & & & 1,00 & \\
\hline 5 a 8 anos & $-0,999$ & 0,04 & 0,37 & $0,14-0,97$ & $-0,159$ & 0,39 & 0,85 & $0,59-1,23$ & 0,243 & 0,31 & 1,28 & $0,79-2,05$ \\
\hline 9all anos & 0,460 & 0,56 & ।,58 & $0,33-7,64$ & 0,859 & 0,01 & 2,36 & $1,16-4,80$ & 0,086 & 0,89 & 1,09 & $0,33-3,56$ \\
\hline 12 ou mais anos & 0,409 & 0,51 & 1,51 & $0,44-5,11$ & 0,096 & 0,74 & 1,10 & $0,62-1,95$ & $-0,168$ & 0,70 & 0,85 & $0,36-1,97$ \\
\hline \multicolumn{13}{|l|}{ Grupo de idade } \\
\hline 60-69 anos & & & 1,00 & & & & 1,00 & & & & 1,00 & \\
\hline $70-79$ anos & 0,774 & 0,00 & 2,17 & I,26-3,75 & $-0,402$ & 0,01 & 0,67 & $0,49-0,91$ & $-0,487$ & 0,03 & 0,61 & $0,40-0,95$ \\
\hline$\geq 80$ anos & 0,769 & 0,03 & 2,16 & $1,04-4,48$ & $-0,671$ & 0,00 & 0,51 & $0,32-0,83$ & $\begin{array}{l}-1,864 \\
-1,10\end{array}$ & 0,00 & 0,16 & $0,05-0,44$ \\
\hline \multicolumn{13}{|l|}{ Problema crônico de saúde } \\
\hline Sim & & & 1,00 & & & & 1,00 & & & & 1,00 & \\
\hline Não & $-0,534$ & 0,03 & 0,59 & $0,35-0,97$ & $-0,485$ & 0,00 & 0,62 & $0,47-0,81$ & $-0,609$ & 0,00 & 0,54 & $0,36-0,81$ \\
\hline \multicolumn{13}{|l|}{ Renda domicliliar } \\
\hline I a $2 S M$ & & & 1,00 & & & & & 1,00 & & & 1,00 & \\
\hline $2 a 5 S M$ & 0,817 & 0,04 & 2,27 & $1,01-5,10$ & 0,937 & 0,00 & 2,55 & I,44-4,54 & 0,609 & 0,15 & 1,84 & $0,80-4,23$ \\
\hline 5 a $105 M$ & 0,115 & 0,80 & 1,12 & $0,44-2,86$ & 0,998 & 0,00 & 2,71 & $1,49-4,93$ & 0,917 & 0,03 & 2,50 & $1,07-5,86$ \\
\hline$\geq 10 \mathrm{SM}$ & $-0,531$ & 0,34 & 0,59 & $0,20-1,76$ & $\mathrm{I}, 273$ & 0,00 & 3,57 & $1,95-6,54$ & 1,192 & 0,00 & 3,29 & I,40-7,76 \\
\hline
\end{tabular}

OB-obesidade, SP-sobrepeso, BP- baixo peso, EU-eutrofia, SM-salário mínino; Coef. - coeficiente

Aqueles com renda domiciliar acima de 10 salários mínimos apresentaram 3,29 vezes mais chance de obesidade $(p=0,00, I C 95 \%$ I,40$7,76)$ e 3,57 vezes mais chance de sobrepeso ( $p=0,00$, IC $95 \%$ 1,95-6,54). Nestas duas categorias de renda domiciliar, não houve diferenças entre eutróficos e portadores de baixo peso ( $p=0,80$ e 0,34 , respectivamente). Já a categoria renda domiciliar entre 2 e 5 salários mínimos não apresentou diferença entre obesos e eutróficos $(p=0,15)$, aumentou em 2,5 vezes a chance de sobrepeso $(p=0,00$, I,44-4,54) e de baixo peso em 2,7 vezes ( $p=0,04$, IC $95 \%$ I, $01-5,10)$.

A categoria de escolaridade tomada como padrão foi a de menos anos completos de estudo - I a 4 anos. A categoria 5 a 8 anos de estudo não apresentou diferença entre idosos obesos e eutróficos $(p=0,3 \mathrm{I})$ e portadores de sobrepeso e eutróficos $(p=0,39)$, diminuindo em 0,37 vez a chance de baixo peso (2,7 vezes mais chance de ser eutrófico, $p=0,04$, IC 95\% 0, I4-0,97). A categoria 9 a II anos de estudo não apresentou diferença entre obesos e eutróficos $(p=0,88)$, aumentou em 2,36 vezes a chance de sobrepeso ( $p=0,01$, IC 95\% I, I6-4,80) e não apresentou diferença entre portadores de baixo peso e eutróficos $(p=0,56)$. A categoria 12 ou mais anos de estudo não apresentou diferença entre os idosos portadores de obesidade, sobrepeso e baixo peso em relação ao eutrófico ( $p=0,69,0,74$ e 0,51, respectivamente).

\section{Discussão}

O envelhecimento da população brasileira é irreversível, existindo um crescente interesse em identificar fatores que levam ao envelhecimento sadio. A boa nutrição durante a vida é um destes fatores. A manutenção de estado nutricional adequado não significa, necessariamente, maior sobrevida, mas interfere positivamente influenciando maior número de pessoas a se aproximarem do seu ciclo máximo de vida².

Dentre os diversos métodos usados para a avaliação nutricional destaca-se a antropometria. $\mathrm{O}$ índice de massa corporal (IMC), uma combinação das medidas de peso e altura, é o parâmetro mais usado para rastreamento de alterações do estado nutricional entre adultos e idosos. Existem críticas relacionadas aos pontos de corte de IMC e a principal delas é que pontos de corte baseados em dados de adultos podem não ser adequados para uso em idosos, devido às mudanças na composição corporal relacionadas ao envelhecimento ${ }^{7}$. Para adultos, a relação entre IMC e mortalidade, quando representada graficamente, é uma curva em forma de $J$ ou de $U$. As causas de mortes nos extremos da curva são diferentes: altas taxas de mortalidade entre indivíduos com baixo IMC são predominantemente devidas a causas digestivas e pulmonares; em indivíduos com altos valores de IMC as causas predominantes são as doenças cardiovasculares, diabetes mellitus e doenças da vesícula biliar. O menor risco de mortalidade fica, aproximadamente, no intervalo de IMC de 20 a $30 \mathrm{~kg} / \mathrm{m}^{2}(2,8)$. Em idosos, o IMC, além de predizer mortalidade e morbidade, está associado com capacidade para viver de forma independente, mobilidade e preservação do estado mental2.

A comparação com estudos brasileiros anteriores mostra que, no intervalo de sete anos entre a PNSN realizada em $1989^{\circ}$ e os dados do presente estudo colhidos pelo IBGE entre 1996/I997, a prevalência de baixo peso diminuiu de $8,1 \%$ para $5,7 \%(p<0,01)$, a prevalência de eutrofia manteve-se estável ( $51,2 \%$ e 50,4\%, $p=0,60)$, a prevalência 
de sobrepeso aumentou (28,5\% para 32,3\%, $p<0,0$ I) e a prevalência de obesidade se estabilizou (I2,3\% para II,6\%, $p=0,46)$. Os resultados encontrados no presente estudo mostram tendência de melhora do perfil nutricional, com diminuição do baixo peso e estabilização da eutrofia e da obesidade entre os idosos brasileiros. Os extremos de classificação nutricional - baixo peso e obesidade - não apresentaram aumento de prevalência nesse período. Houve, entretanto, aumento da prevalência de sobrepeso.

Considerando a distribuiçãao por gênero, observa-se diminuição da prevalência de baixo peso entre mulheres $(p<0,01)$ e tendência de sua diminuição entre homens idosos $(p=0, \mid 3)$; aumento da prevalência de eutrofia entre mulheres $(p=0,03)$ e diminuição entre homens idosos $(p=0,01)$; aumento da prevalência de sobrepeso entre homens $(p<0,0$ I $)$ e estabilização dos valores entre mulheres idosas $(p=0,72)$; estabilização da prevalência de obesidade entre homens $(p=0,89)$ e mulheres idosas $(p=0,22)$.

\section{Baixo peso}

Apesar da prevalência de baixo peso encontrada neste estudo ser considerada baixa pelos critérios da OMS², a comparação com estudos de países europeus e EUA $A^{10,11,12}$ mostra que ela ainda é alta, possivelmente porque a transição nutricional está em fase mais avançada nos países desenvolvidos, refletindo maior desenvolvimento socioeconômico.

Neste estudo, os idosos com cinco a oito anos de escolaridade apresentaram menor chance de baixo peso comparados aos com quatro ou menos anos de estudo. Esta associação pode estar sofrendo influência do nível socioeconômico normalmente associado com escolaridade ${ }^{13}$. Porém, ainda no presente estudo, observa-se que os idosos com renda domiciliar entre 2 e 5 salários mínimos apresentam chance 2,27 vezes maior de apresentar baixo peso quando comparados àqueles com renda domiciliar menor que 2 salários mínimos. Dados da PNSN associam baixa renda com precárias condições de vida e baixo peso na população idosa. A elevação da renda, da escolaridade, da qualidade da moradia e maior acesso a bens e serviços reduziram até mesmo eliminando o baixo peso nos grupos sociais privilegiados? ${ }^{9}$. As condições socioeconômicas desempenham papel fundamental em relação à qualidade de vida na velhice. Renda suficiente para atendimento das necessidades básicas, bom nível de escolaridade, relações familiares adequadas, boas condições de sociabilidade e atividade produtiva podem contribuir para maior autonomia do idoso ${ }^{4}$.

Outra variável associada de maneira independente com baixo peso é a faixa etária. Os idosos com maior idade têm chance maior de apresentar baixo peso. $O$ envelhecimento promove mudanças importantes na massa muscular e no padrão de distribuição de gordura

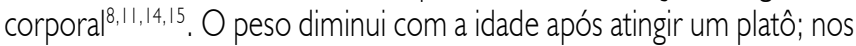
homens ao redor de 65 anos, nas mulheres cerca de 10 anos mais tarde ${ }^{2}$.

Os idosos portadores de problema crônico de saúde apresentaram maior chance de baixo peso. $\mathrm{Na}$ Tabela 3 , o resultado do OR indica que a chance do idoso que não tem problema crônico de saúde apresentar baixo peso é 0,59 daquela do idoso com problema crônico de saúde. Invertendo-se a ordem das categorias, observa-se que a chance do idoso com problema crônico de saúde apresentar baixo peso é 1,69 vez maior. Algumas possíveis explicações para esta associação podem ser alteração da absorção e do metabolismo ou consumo pela própria doença crônica.

Diferentemente do relatado na literatura", no presente estudo o gênero não se associa de maneira independente com baixo peso, mas somente com sobrepeso e obesidade.

\section{Sobrepeso}

A maior prevalência de sobrepeso foi encontrada, neste trabalho, entre idosos do sexo feminino, sendo esta uma das variáveis associadas de forma independente (análise multivariada) com o desvio do estado nutricional com chance $(\mathrm{OR})$ 1,32 vez maior (Tabela 3). Outros estudos não quantificaram a chance $(\mathrm{OR})$, mas apresentaram valores de médias de IMC maiores no sexo feminino do que no masculino ${ }^{16-19}$. Uma possível explicação para a maior chance das mulheres apresentarem sobrepeso ou obesidade é o maior acúmulo de gordura visceral, diferenças na ingestão alimentar e maior expectativa de vida. Além disso, a menopausa é acompanhada por aumento de peso e adiposidade. Há, durante o processo de envelhecimento, redistribuição progressiva da gordura com diminuição do panículo adiposo subcutâneo dos membros e acúmulo intra-abdominal. As mulheres acumulam mais gordura subcutânea que os homens e a perdem em idades mais tardias ${ }^{2}$, mas tendem a trocar seu padrão de dieta e a praticar exercícios mais rapidamente que os homens.

No presente estudo, o idoso que tem 9 a I I anos de escolaridade tem chance 2,36 vezes maior de apresentar sobrepeso quando comparado ao idoso com 4 ou menos anos de escolaridade (Tabela 3). Esta associação pode refletir influência do nível socioeconômico, apesar desta variável associar-se de maneira independente com sobrepeso. Observa-se, usando o grupo de idosos com renda domiciliar até 2 salários mínimos como padrão, que o aumento da chance de sobrepeso é proporcional ao aumento da renda domiciliar: 2,55, 2,77 e 3,57, respectivamente, para os grupos com renda domiciliar entre 2 e 5, 5 a 10 e maior que 10 salários mínimos.

Outra variável associada de maneira independente com sobrepeso foi a faixa etária, indicando que o idoso mais velho possui chance menor de apresentar sobrepeso. Esta, possivelmente, associa-se com as mudanças na composição corporal citadas anteriormente e também observadas entre os idosos italianos" ${ }^{\prime \prime}$.

$\mathrm{Na}$ Tabela 3, o resultado do OR indica que a chance do idoso que não tem problema crônico de saúde apresentar sobrepeso é 0,62 a chance do idoso portador de problema crônico. Invertendo-se a ordem das categorias, observa-se que a chance do idoso portador de problema crônico de saúde apresentar sobrepeso é I,6I vez maior que a do idoso saudável. Portanto, o idoso portador de problema crônico de saúde tem maior chance de apresentar sobrepeso.

\section{Obesidade}

Neste trabalho, as idosas apresentam chance 4, I I vezes maior de obesidade (Tabela 3). Na Itália, esta chance é de 2,2" e, em Bambuí, $3,1^{20}$. Na PNSN, a prevalência de obesidade foi de $5,7 \%$ entre homens e $18,2 \%$ entre mulheres ${ }^{9}$. A prevalência de obesidade entre idosos europeus, americanos, mexicanos e venezuelanos é maior do que a 
observada no presente estudo, com maior proporção entre as idosas ${ }^{10,11,16,18,21}$. Outros estudos brasileiros também observaram o mesmo comportamento em relação ao gênero ${ }^{20,22}$.

A comparação entre a PNSN e a PPV mostra tendência da manutenção da prevalência de obesidade entre homens e mulheres idosas. Este fato é de grande importância desde que a obesidade, mais do que o sobrepeso, associa-se a várias doenças crônico-degenerativas, aumentando a morbi-mortalidade e o impacto sobre o sistema de saúde. Ainda neste estudo, os idosos com idade mais avançada apresentaram menor chance de obesidade e os portadores de problema crônico de saúde maior chance de obesidade.

No estudo Canada Fitness Survey, foi descrita relação significativa, em forma de curva de I para homens e linear para mulheres, entre IMC e taxas de mortalidade por todas as causas ${ }^{23}$. No The Rotterdam Study, foi descrito que a relação entre IMC e mortalidade parece ser menos pronunciada em idosos do que entre adultos jovens ${ }^{7}$. As prováveis explicações podem ser a sobrevivência seletiva dos idosos, a mais alta taxa de mortalidade neste grupo e a diminuição da massa muscular e da altura que ocorrem com a idade.

Como no sobrepeso, a chance de obesidade aumenta com a elevação da renda domiciliar. Esta diferença é estatisticamente significativa comparando-se os grupos com renda domiciliar entre 5 a 10 $(\mathrm{OR}=2,50)$ e maior que $10(\mathrm{OR}=3,29)$ salários mínimos ao grupo com renda domiciliar até 2 salários mínimos. Outro estudo, também com dados da PPV, mostrou que, entre adultos, a obesidade em mulheres associa-se negativamente com educação e, apenas no Nordeste, positivamente com renda; entre os homens, associa-se positivamente com renda mas não com educação $0^{5}$. Neste estudo, a obesidade não associou-se significativa e independentemente com escolaridade, o que justifica a necessidade de estudos específicos para a população idosa.

A comparação dos dados de adultos dos três inquéritos nutricionais brasileiros demonstra que a associação entre renda e sobrepeso/ obesidade permaneceu positiva em todos os grupos de idade, regiões e grupos de renda, com exceção das mulheres adultas do Sudeste ${ }^{5}$. Neste grupo de mulheres, a associação positiva entre renda e obesidade verificada em 1975 foi revertida em 1997 para uma associação negativa significante. Um dos fatores responsáveis pelo declínio da obesidade entre essas mulheres foi a promoção de prática de atividade física e de hábitos alimentares saudáveis transmitidos, principalmente, pelos veículos de comunicação de massa brasileiros ${ }^{5}$. A adesão aos programas de promoção de hábitos de vida saudáveis pode ter influenciado o controle do excesso de peso. A mesma estratégia pode ser utilizada em idosos.

Os achados do presente estudo mostram que o excesso de peso, e não a desnutrição, constitui o principal problema nutricional da população idosa brasileira.

\section{Conclusão}

O perfil nutricional dos idosos é caracterizado pela alta prevalência de eutrofia e sobrepeso e pequena prevalência de baixo peso e obesidade. $O$ gênero feminino tem maior risco de sobrepeso e obesidade. A escolaridade aumenta o risco de sobrepeso e diminui o de baixo peso, assim como a renda familiar, que aumentou a chance de sobrepeso e obesidade. Idoso saudável (não portador de problema crônico de saúde) tem menor chance de apresentar desvios do estado nutricional. Idosos com idade mais avançada apresentam maior chance de baixo peso e menor chance de sobrepeso e obesidade.

\section{Conflito de interesse: não há}

\section{SUMMARY}

\section{Nutritional STATUS AND RELATED FACTORS AMONG ELDERLY BraZiLIaNS}

OBIECTNE. This study describes the nutritional status and associated factors among elderly Brazilians.

Methods. Data from "Pesquisa sobre Padrões de Vida" (PPV- Life Pattern Research), conducted by the "Brazilian Geographic and Statistics Institute" in 1996/1997 were used. Body Mass Index (BMI) was applied to classify nutritional status and is the response variable of this study. Multivariate analysis with polytomous logistic regression was used.

RESULTS. Prevalence of underweight was 5.7\%, eutrophy 50.4\%, overweight $32.3 \%$ and obesity $11.6 \%$. Women have a 1.32 greater chance of overweight (Cl 95\% 0.99-1.74; $p=0.05$ ) and 4.1 I of obesity (CI 95\% 2.57-6.57; $p<0.01$ ). Higher age increased risk of underweight and diminished that of overweight and obesity. Elderly with a lower income have a higher risk of underweight and those with a greater income have higher risk of overweight and obesity. Elderly with a chronic disease have a higher risk of changes in the nutritional status.

CONCLUSION. Results show a higher prevalence of overweight and eutrophy and a lower prevalence of obesity and underweight. Characteristics associated with nutritional status other than eutrophy are: gender, schooling, income, chronichealth problem and age. [RevAssoc Med Bras 2006; 52(4): 2|4-2I]

KEY WORDS: Nutritional surveys. Epidemiological profiles. Body weight. Elderly.

\section{ReferênCIAS}

I. Chaimowicz F. A Saúde dos idosos brasileiros às vésperas do século XXI: problemas, projeções e alternativas. Rev Saúde Pública | 997;3 I (2): I 84-200.

2. Who Expert Committee on Physical Status: the use and interpretation of antropometry physical status: the use and interpretation of antropometry: report of a WHO Expert Commitee. Switzerland; 1995.

3. Coelho AK, Fausto MA. Avaliação pelo nutricionista. In: Maciel A. Avaliação multidisciplinar do paciente geriátrico. Rio de Janeiro: Revinter; 2002.

4. Pesquisa sobre padrões de vida 1996-1997. Ministério do Planejamento, Orçamento e Gestão, Instituto Brasileiro de Geografia e Estatística, $2^{\mathrm{a}}$ ed. Rio de Janeiro: IBGE; 1999.

5. Monteiro CA, Conde WL, Popkin BM. Symposium: Obesity in Developing Countries: Biological and Ecological Factors. Independent Effects of Income and Education on the Risk of Obesity in the Brazilian Adult Population. J Nutr 200 I; I I (3):88 I S-6S.

6. Monteiro CA, Benício MHDA \& Popkin BM. Economic and culturaleducation predictors of overweight in urban and rural brazilian women. Rev Bras Nutr Clin 2000; I 5(2):253-60.

7. Visscher TL, Seidell JC, Molarius A, Kuip D, Hofman A, Witteman JCM. A comparison of body mass index, waist-hip ratio and waist circumference as predictors of all-cause mortality among the elderly: the Rotterdam study. Int J Obes 200 I;25(I0): | 730-5. 
8. Anjos, LA. Índice de massa corporal como indicador do estado nutricional de adultos: revisão da literatura. Rev Saúde Pública 1992;26(6):43 I-6.

9. Tavares EL, Anjos LA. Perfil antropométrico da população idosa brasileira. Resultados da Pesquisa Nacional sobre Saúde e Nutrição. Cad Saúde Pública 1999; 1 5(4):759-68.

10. de Groot CPGM, Enzi G, Perdigão AL, Deurenberg P. Longitudinal changes in the anthropometric characteristics of elderly Europeans. SENECA Investigators. Eur J Clin Nutr 1996;50(Suppl 2):S9-SI 5.

II. Perissinoto E, Pisent C, Sergi G, Grigoletto F, Enzig E. Anthropometric measurements in the elderly: age and gender differences. Br J Nutr 2002;87(2): 177-86.

12. Kuczsmarski MF, Kuczsmarski RJ, Najjar M. Descriptive anthropometric reference data for older americans. J Am Diet Assoc 2000; I 00( I ):59-66.

13. Monteiro CA, Conde WL, Castro IRR. The changing relationship between education and risk of obesity in Brazil (1975-1997). Cad Saúde Pública 2003; I 9(Supl I):67-75.

14. Seidell JC, Visscher TLS. Body weigth and weigth change and their health implications for the elderly. Eur J Clin Nutr 2000;54(Suppl 3):S33-S9.

15. Omran ML, Salem P. Diagnosing undernutrition. Clin Geriatr Med 2002; | 8(7):7| 9-36.

16. Yearick ES. Nutrition Status of the elderly: anthropometric and clinical findings. J Gerontol 1978;33(5):657-62.

17. Chilima DM, Ismail SJ. Anthropometrics characteristics of older people in rural Malawi. Eur J Clin Nutr 1998;52(5):643-9.
18. Velázquez-Alva Mc, Castilho-Martínez L, Irigoyen-Camacho E, ZepedaZepeda MA, Gutiérrez-Robledo LM, Cisneros-Moysen P. Estudio antropométrico en un grupo de hombres y mujeres de la tercera edad en la ciudad de México. Salud Publica Mex 1996;(38):466-74.

19. Cabrera MAS, Jacob Filho W. Obesidade em idosos: prevalência, distribuição e associação com hábitos e co-morbidades. Arq Bras Endocrinol Metab 200 I;45(4):494-50 I.

20. Barreto SM, Passos VMA, Lima-Costa MFF. Obesidade e baixo peso entre idosos brasileiros. Projeto Bambuí. Cad Saúde Pública 2003; I 9(2):60512.

21. Falque-Madrid L, Piñero-Corredor M, Rodriguez NZ, Quintero J, Gabarrón AS, Arias-Marquez N. Estado nutricional y composición corporal de un grupo de adultos no institucionalizados del Estado Zulia, Venezuela. Arch Latinoam Nutr 1996;46(3): 190-5.

22. Gigante DP; Barros FC; Post CLA; Olinto MTA. Prevalência de obesidade em adultos e seus fatores de risco. Rev Saúde Pública I997;3 I (3):236-46.

23. Katzmarzyk PT, Craig CL, Bouchard C. Adiposity, adipose tissue distribution and mortality rates in the Canada Fitness Survey follow-up study. Int J Obes 2002;26( I 0): 1054-9.

Artigo recebido: 28/02/05

Aceito para publicação: 07// I/05 